\title{
Failure to Thrive with Polyuria
}

\section{Molly Band* and Cynthia D'Alessandri-Silva}

Department of Nephrology, Connecticut Children's Medical Center, USA

*Corresponding author: Molly Band, Director, Department of Nephrology, Connecticut Children's Medical Center, 282 Washington St, Hartford, CT 06106, USA, Tel: 860-545-9395; Fax: 860-545-8422; E-mail: mband@connecticutchildrens.org

Rec Date: July 24, 2017, Acc Date: August 16, 2017, Pub Date: August 18, 2017

Citation: Band M, D’Alessandri-Silva C (2017) Failure to Thrive with Polyuria. Med Case Rep. Vol.3 No.4: 34.

\section{Abstract}

Polyuria is excessive or an abnormally large production or passage of urine. Frequent urination is usually an accompanying symptom. Polyuria often appears in conjunction with polydipsia (increased thirst). The most common cause of polyuria in both adults and children is uncontrolled diabetes mellitus, which causes osmotic diuresis, when glucose levels are so high that glucose is excreted in the urine. This case report discuses about a seven-month-old male patient with failure to thrive and polyuria in the setting of known sickle cell disease.

Keywords: Polyuria; Fanconi syndrome; Acidosis; Failure to thrive

Abbreviations: HEENT: Head, Ears, Eyes, Nose and Throat; kg: Kilogram; BUN: Blood Urea Nitrogen; $\mathrm{CO}_{2}$ : Carbon dioxide; IV: Intravenous; GT: Gastrostomy Tube; ESRD: End Stage Renal Disease

\section{Introduction}

A seven-month-old male presents with failure to thrive and polyuria in the setting of known sickle cell disease. His exam was notable for frontal bossing, gross motor delay and palpable beading along his chest wall. Initial laboratory works up revealed acidemia and nephrology was consulted. Pertinent labs were bicarbonate of $11 \mathrm{mmol} / \mathrm{L}$, phosphorus of $1.9 \mathrm{mg} / \mathrm{dl}$ and a venous $\mathrm{pH}$ of 7.26. The urine specific gravity was 1.006 with $2000 \mathrm{mg} / \mathrm{dL}$ glucose and $30 \mathrm{mg} / \mathrm{dL}$ of protein. Quantitative urine amino acid screen was positive for multiple important amino acids. A chest $\mathrm{x}$-ray revealed prominence of the anterior ribs and rickets was diagnosed. The renal ultrasound revealed echogenic renal pyramids bilaterally. Fanconi syndrome was suspected and a wide differential diagnosis was considered. Ultimately, we present a case of infantile nephropathic cystinosis in a child with $\mathrm{Hgb}-\mathrm{SS}$ disease. It is important to have a wide differential for children with rickets as this is an unusual presentation given most children with cystinosis are classically fair with blonde hair.

\section{Case Presentation}

We present a seven-month old Somalian male with a history of Sickle Cell Disease Hgb-SS who presented with a two to three-week history of upper respiratory symptoms. History was also positive for cough, nasal congestion, decreased oral intake, and poor weight gain. He had been taking about one ounce of formula every two to three hours as well as two to three ounces of free water. He reportedly had been making two or three wet diapers per day with normal appearing stools. Review of his growth chart revealed a normal birth weight at 2.8 kilograms $(\mathrm{kg})$ and adequate weight gain for the first four months of life, following between the $15^{\text {th }}$ and $35^{\text {th }}$ percentile for weight and length for age. At four months of age, his weight percentile dropped to the $8.7^{\text {th }}$ percentile and length was stable at the $21.3^{\text {rd }}$ percentile for age. At presentation, his weight was at the $0.28^{\text {th }}$ percentile and length was at the $15^{\text {th }}$ percentile for age.

Physical examination on presentation revealed a small for age seven-month-old male with nearly closed anterior fontanelle and prominent forehead. Head, eyes, ears, nose, and throat (HEENT) examination revealed no periorbital edema with nasal mucosa edema and rhinorrhea. Chest examination revealed heart with regular rate and rhythm, lungs with transmitted upper airway sounds, and palpable beading along the anterolateral aspect of the chest. Abdominal exam revealed a palpable liver edge without splenomegaly. Abdomen was otherwise soft with normal bowel sounds. Musculoskeletal examination revealed poor muscle tone and gross motor delays without achievement of independent sitting.

Laboratory workup at presentation revealed a blood urea nitrogen (BUN) of $2 \mathrm{mg} / \mathrm{dL}(4-14 \mathrm{mg} / \mathrm{dL})$, creatinine of 0.2 $\mathrm{mg} / \mathrm{dL}(0.2-0.4 \mathrm{mg} / \mathrm{dL})$, sodium of $138 \mathrm{mmol} / \mathrm{L}$, potassium of $3.5, \mathrm{mmol} / \mathrm{L}$ chloride of $111 \mathrm{mmol} / \mathrm{L}$, low carbon dioxide $\left(\mathrm{CO}_{2}\right)$ of $11 \mathrm{mmol} / \mathrm{L}$, and a low phosphorus of $1.9 \mathrm{mg} / \mathrm{dL}$ (4.0-7.8 $\mathrm{mg} / \mathrm{dL}$ ). His hemoglobin and hematocrit were low at $7.9 \mathrm{~g} / \mathrm{dL}$ $(11.0-16.3 \mathrm{~g} / \mathrm{dL})$ and $25.5 \%(31.5 \%$ to $48.9 \%)$ respectively. His initial treatment included hydration with intravenous (IV) fluids and he was placed on a strict feeding regimen to meet caloric goals for catch up growth. Despite these interventions, he had a persistent acidemia with serum bicarbonate levels ranging from $11-13 \mathrm{mmol} / \mathrm{L}$. Venous blood gas revealed a venous $\mathrm{pH}$ of 7.26 (7.33-7.43) and a total venous $\mathrm{CO}_{2}$ of 15 $\mathrm{mmol} / \mathrm{L}(23-29 \mathrm{mmol} / \mathrm{L})$. 
During his work up for failure to thrive and acidosis, his urinalysis revealed a specific gravity of 1.006 with $2000 \mathrm{mg} / \mathrm{dL}$ glucose and $30 \mathrm{mg} / \mathrm{dL}$ of protein. Polyuria was noted with a urine output measuring $7.5 \mathrm{cc} / \mathrm{kg} / \mathrm{hr}$. Thyroid studies were normal. Further urine studies revealed significant levels of low molecular weight proteinuria with $46.3 \%$ albumin and $53.7 \%$ globulin on urine electrophoresis. Urine beta-2 microglobulin was considerably elevated at $530,000 \mathrm{mcg} / \mathrm{g}$ creatinine. Quantitative urine amino acid screen revealed multiple metabolically important amino acids that were mildly to markedly increase.

The differential diagnosis of proximal renal tubular acidosis in a child includes idiopathic, inherited, and acquired causes. This combined with the constellation of polyuria, hypokalemia and glycosuria should cause suspicion for Fanconi syndrome. Inherited causes include cystinosis, tyrosinemia, galactosemia, fructosemia, glycogen storage disease, Wilson's disease, and Lowe's syndrome. Acquired causes include medications such as aminoglycosides, exposure to heavy metals such as lead, or Vitamin D deficiency. With concerns for proximal renal tubular acidosis and Fanconi syndrome, a renal ultrasound was performed that revealed echogenic renal parenchyma bilaterally. A chest $x$-ray revealed a slight bulbous prominence to several of the lower anterior ribs bilaterally suggestive of rickets.

With the patient's multisystem involvement, Infantile Nephropathic Cystinosis was suspected. He was found to have elevated cysteine levels in his peripheral blood leukocytes as a confirmatory test to diagnose Cystinosis. The patient was started on high doses of bicarbonate at $20 \mathrm{mEq}$ per kilogram per day, phosphorus supplementation, vitamin D, as well as oral cysteamine and cysteamine eye drops. He required gastrostomy tube (GT) placement for enteral feeds for weight gain and growth.

\section{Discussion}

Cystinosis is a rare autosomal recessive disorder caused by mutations in the CTNS gene (17p13.2) [1-7]. It is a lysosomal storage disorder whereby there is a defect in the membrane transport protein, cystinosin, causing systemic accumulation of cystine crystals and is the most common cause of inherited renal Fanconi syndrome in children $[1,3,4,5]$.

The incidence of cystinosis is estimated to be between 1 per 100,000 and 200,000 live births [2-5], although the incidence rates may vary, particularly in selected populations with founder mutations such as Brittan and France [1,3]. The incidence rates are higher in communities and families affected by consanguinity [1].

There are three distinguishable forms of cystinosis that are based on age of presentation and extent of renal involvement. Infantile nephropathic cystinosis, the most common and severe form, affects about $95 \%$ of patients with cystinosis [1-3]. The other two forms of cystinosis are juvenile cystinosis and non-nephropathic ocular cystinosis. Typically, patients with juvenile cystinosis are diagnosed later in childhood. These patients have a slower progression toward end stage renal disease (ESRD). The least common form of cystinosis, ocular cystinosis, affects only the eyes with corneal accumulation of cystine crystals causing photophobia $[1,2,5]$. The focus of this discussion with be infantile nephropathic cystinosis.

Because of its rarity, the diagnosis of cystinosis is often delayed [2]. Patients are typically asymptomatic until 6-12 months of age, at which time they present with non-specific findings of growth failure, vomiting, polyuria, and dehydration $[1,5]$. Much like the patient we presented, patients with cystinosis typically have normal birth weight and lengths with growth retardation beginning around 6-12 months of age [1]. Further testing reveals significant evidence of proximal tubule dysfunction. Laboratory investigation typically reveals serum electrolyte abnormalities including hypokalemia, hypophosphatemia, metabolic acidosis, and hypocalcemia. Patients also have urinary loss of amino acids, sodium, potassium, bicarbonate, magnesium, carnitine, calcium, phosphate, glucose, and low molecular weight proteins [1-3]. Patients can also have vitamin D resistant hypophosphatemic rickets [1-4]. Serum creatinine is typically normal at presentation, but if left untreated or if treatment is delayed to early childhood, infantile nephropathic cystinosis will lead to renal failure within the first ten years of life $[1,4]$.

There are three means for confirming the diagnosis of cystinosis [1]. Primarily cystinosis is confirmed by detection of elevated cystine levels in white blood cells. This requires a dedicated laboratory. Additional testing includes genetic analysis of CTNS gene and slit lamp exam demonstrating corneal cysteine crystal accumulation [1-4]. It is important to note that corneal crystals are typically not visualized on slit lamp ophthalmologic exam until between one and two years of age [1-3]. In developing nations where other means of diagnosis are not as widely available, this may delay diagnosis [1]. Newborn screening as not yet been developed [1].

Although rare, cystinosis fortunately has disease-specific treatment and is the only lysosomal storage disease treatable with cystine-depleting therapy [1]. Oral cysteamine, an aminothiol, depletes lysosomal cystine in the body, delaying progression to end stage renal disease by several years. Immediate release cysteamine therapy is administered every 6 hours around the clock and must be continued throughout the patient's lifetime. The dose is based on body surface area and adjusted based on the trend of the leukocyte cystine level $[1,5]$. The side effects of oral cysteamine include nausea; often times requiring proton pump inhibitors and/or a Nissen fundoplication [1].

Oral cysteamine also helps ameliorate many extra-renal manifestations of cystinosis including preventing growth retardation if started in early infancy, although does not affect tubular dysfunction or reduce corneal cystine accumulation. For this, topical cysteamine is used to dissolve the corneal cystine crystals $[1,4,5]$, and must be used frequently throughout the day, often resulting in poor compliance. As expected, poor compliance with both oral cysteamine and topical cysteamine has a detrimental effect, with more rapidly progressive renal insufficiency and poor growth [1,7]. 
Management of the organ complications of cystinosis is a large piece to the treatment, in addition to targeted cystinedepletion therapy $[1,2]$. Symptomatic treatment includes maintaining adequate fluid, electrolyte, and vitamin supplementation, as well as appropriate nutrition to support catch up growth. Supplementation often includes sodium, potassium, bicarbonate, phosphorus, and 1-25 (OH $)_{2}$ cholecalciferol $[1,3,4]$. Due to growth failure accompanied by poor appetite and vomiting, children most often require early gastric tube placement [2,3]. With the multiple endocrinopathies that accompany cystinosis, hormonal therapy may be necessary. This includes thyroid hormone, growth hormone, and testosterone therapy [1].

\section{Conclusion}

In the event of end stage renal disease (ESRD), renal transplantation is the treatment of choice. Most often, native nephrectomy is not required, but may be considered based on the degree of polyuria. Although renal transplantation is a cure for the renal component of cystinosis, it does not affect the extra-renal complications. Because of this, cysteine-depleting therapy must be continued. Graft survival is reported to be superior to that of other renal diseases [1-3].

\section{Declarations}

\section{Ethics approval and consent to participate}

Not applicable.

\section{Consent for publication}

This can be provided upon request.

\section{Availability of data and material}

Not applicable.

\section{Competing interests}

The authors declare that they have no competing interests.

\section{Funding}

Not applicable.

\section{Authors" contributions}

MB compiled the patient data, researched review articles, drafted the initial manuscript, reviewed and revised the manuscript, and approved the final manuscript as submitted.

CDS reviewed articles and revised the manuscript, and approved the final manuscript as submitted.

\section{References}

1. Elonem MA, Veys KR, Soliman NA, Van Dyck M, Van Den Huevel LP, et al. (2016) Cystinosis: A review. Orphanet J Rare Dis 11: 47.

2. Emma F, Nesterova G, Langman C, Labbe A, Cherqui $S$, et al. (2014) Nephropathic cystinosis: An International Consensus Document. Nephrol Dial Transplant 29: iv87-iv94.

3. Nesterova G, Gahl WA (2013) Cystinosis: The evolution of a treatable disease. Pediatr Nephrol 28: 51-59.

4. Gahl WA, Thoene JS, Schneider JA (2002) Cystinosis. N Engl J Med 347: 111-121.

5. Wilmer MJ, Emma F, Levtchenko EN (2010) The pathogenesis of cystinosis: Mechanisms beyond cystine accumulation. Am J Renal Physiol 299: F905-F9016.

6. Mahoney CP, Striker GE (2002) Early development of the renal lesions in infantile cystinosis. Pediatr Nephrol 15: 50-56.

7. Langman CB, Greenbaum LA, Sarwal M, Grimm P, Niadet $P$, et al. (2012) A randomized controlled crossover trial with delayedrelease cysteamine bitrate in nephropathic cystinosis: Effectiveness on white blood cell cystine levels and comparison study. Clin J Am Soc Nephrol 7: 1112-1120. 